\title{
Quem se Beneficia dos Programas de Ginástica Laboral?
}

\author{
Regina Heloisa Maciel ${ }^{1}$, Ana Maria F. Costa Albuquerque ${ }^{2}$, \\ Adriana C. Melzer ${ }^{2}$ e Suzete Rodrigues Leônidas ${ }^{2}$
}

Universidade Federal do Ceará

\begin{abstract}
O problema das LER/DORT tem ocupado grande parte dos estudos relacionados à saúde dos trabalhadores. Suas causas e conseqüências são relativamente conhecidas apesar de ainda haverem controvérsias quanto ao seu diagnóstico e tratamento. A ergonomia tem sido uma das técnicas efetivas na prevenção das LER/DORT por abordar diretamente as condições de trabalho, causas do problema. No entanto, um outro método tem crescido no Brasil na tentativa de prevenir as LER/DORT: os programas de ginástica laboral. Neste trabalho descrevem-se as principais características dos programas de ginástica laboral e discute-se a sua eficácia na prevenção das LER/DORT, bem como outros fatores associados com a implantação desses programas. Conclui-se que, ao contrário do propalado pelas empresas que oferecem serviços de ginástica laboral, ela não é um método ergonômico de prevenção, pois os objetivos e meios são diferenciados. Por outro lado, a ginástica laboral, até o momento, não fez jus à suposição de sua eficácia na prevenção das LER/DORT. Além disso, os programas estão mais a serviço das empresas do que da saúde do trabalhador.
\end{abstract}

Palavras-chave: Ginástica laboral, Ergonomia, Ler/Dort, Prevenção, Saúde ocupacional.

Exercises in the workplace: beneficial to whom?

RSI is a generalized problem and it has been the aim of various studies in the occupational health area. RSI's causes and consequences are relatively known, although the existence of some controversies relating to its diagnoses and prevention. The use of ergonomics techniques have proven effective in RSI management and prevention due mainly to its direct approach to working conditions, known to be linked to its cause. However, another method is increasing in Brazil in an attempt to prevent RSI: exercises in the workplace. This article describes the main characteristics of the workplace exercises programs and discusses its success in the prevention of RSI, as well as other factors related to its implementation in the workplace. The conclusion is that, contrary to the idea disseminated by companies that offer workplace exercises programs; these programs can not be considered ergonomics RSI prevention methods, as the objectives and techniques of the two kinds of interventions are completely different. On the other hand, workplace exercises programs, until the moment, did not provide evidence for the supposition of its effectiveness in RSI's prevention. Besides that, it seems that the programs are more at the enterprises service than to the workers.

Keywords: Workplace exercises, Ergonomics, RSI, Prevention, Occupational health.

\section{O Problema das LER/DORT}

$\mathrm{N}$ os últimos 20 anos, o mundo tem assistido ao progressivo crescimento das Lesões por Esforços Repetitivos/Distúrbios Osteomusculares Relacionados ao Trabalho (LER/DORT), uma síndrome polêmica de dimensões sociais e econômicas, refletida na incapacidade do trabalhador para exercer suas atividades e o sofrimento decorrente disso, bem como a geração de custos significativos para as organizações e o Estado.

Com o avanço das LER/DORT, pesquisadores e profissionais de diversas áreas têm procurado discutir as inúmeras controvérsias que o fenômeno suscita. A primeira controvérsia diz respeito ao próprio diagnóstico, difícil de ser feito e acordado entre os médicos (Verthein \& Gomez, 2001). A segunda controvérsia tem a ver com a tentativa de encontrar possíveis soluções para o problema, que atinge ampla magnitude e proporções epidêmicas. No Brasil, não existem estatísticas sólidas que permitam traçar um quadro geral

1 Prof. Dra. do Mestrado em Saúde Pública.

2 Alunas do Curso de Mestrado em Saúde Pública. 
das LER/DORT, prevalecendo, segundo Souza (1999), os estudos de demandas de serviços de assistência à saúde do trabalhador, estudos de casos entre trabalhadores adoecidos e de casos notificados pelas CATs. De acordo com o Ministério da Saúde (2000) e os dados do Instituto Nacional do Seguro Social/Ministério da Previdência e Assistência Social de 1997, as LER/DORT têm sido, nos últimos anos, a mais prevalente dentre as doenças ocupacionais registradas entre a população trabalhadora segurada.

As causas das LER/DORT são apontadas por diversos pesquisadores como um conjunto de fatores físicos e organizacionais do trabalho que, combinados, possibilitam o surgimento da síndrome. Dentre esses fatores, são citados: posturas inadequadas, natureza e repetitividade de movimentos e aplicação de forças, que podem influenciar diretamente no sistema músculo-esquelético do trabalhador. Os fatores causais indiretos relacionam-se ao conteúdo das atividades e à qualidade da comunicação, períodos prolongados de trabalho, ausência de pausas, não rotatividade de tarefas e fatores psicológicos, tais como o estresse, pressão pela produção e o relacionamento entre chefias e funcionários (Maciel, 1998; Codo, 1998; Ranney, 2000).

A ergonomia tem desempenhado um papel central na prevenção dessas afecções, pois permite a avaliação das condições e ambientes de trabalho, propostas e implementação de soluções técnicas (relacionadas a mudanças nos equipamentos e ambientes físicos) e administrativas (relacionadas à programação de pausas e rodízios e mudanças na organização e conteúdo das atividades), assim reduzindo a freqüência das doenças, os custos financeiros com indenizações e o sofrimento dos trabalhadores (Rios, 1998; Lampl, Wooley, Conine, Hoehne \& Moorman, 2000).

Por outro lado, programas de ginástica laboral têm sido implementados com grande freqüência nas empresas como um método de prevenção das LER/DORT. O objetivo deste trabalho é rever as principais evidências sobre a efetividade da ginástica laboral na prevenção das afecções que compõem a síndrome.

\section{Distinção entre Atividade Física e Ginástica Laboral}

É importante fazer a distinção entre atividade física no local de trabalho ou fora dele e ginástica laboral (GL), pois essas duas práticas têm objetivos diversos e diferem significativamente nos meios e instrumentos que utilizam. Os programas de atividade física consistem em incentivos à prática de esportes ou atividades que levem a um maior dispêndio energético e movimentação da musculatura. Em geral, recomenda-se a prática de um esporte ou atividade física pelo menos três vezes na semana, com uma duração de aproximadamente uma hora por sessão. Para isso existem as academias e outros centros esportivos, em geral, fora dos locais de trabalho. A ginástica laboral tem por objetivo principal a prevenção de doenças ocupacionais, é realizada nos locais de trabalho, três vezes por semana, ou diariamente, por períodos que variam de 8 a 12 minutos, durante a jornada de trabalho.

\subsection{Eficácia dos Programas de Incentivo à Prática de Atividades Físicas}

A atividade física é considerada como benéfica para a saúde física e mental em geral. Assim, vários programas governamentais e comunitários têm sido implantados com o objetivo de incentivar a prática de atividades físicas recreativas, com o intuito de prevenir algumas doenças degenerativas, principalmente as doenças coronárias, hipertensão e estresse. Um 
exemplo é o Programa "Healthy People 2010" (Pessoas Saudáveis 2010) dos Estados Unidos, cujo objetivo é melhorar a saúde e a qualidade de vida através da atividade física diária e que se propõe a várias intervenções para incentivar essa prática. Entre estas intervenções estão ações que atingem as pessoas em seus ambientes de trabalho (Center for Disease Control and Prevention, 2004). Programas semelhantes têm sido desenvolvidos em vários outros países (Titze, Martin, Seiler, Stronneger \& Marti, 2001; Craig, Brownson, Cragg \& Dunn, 2002; Puska, Helasoja, Prätälä, Kasmel \& Klumbiene, 2003; Yancey, et al., 2004). Quando são realizadas ações no ambiente de trabalho, elas procuram verificar a quantidade e a qualidade da prática de atividades físicas dos empregados, em geral, em funções sedentárias, e incentivá-los a praticar esportes, andar a pé ou de bicicleta, dançar e outras atividades que possam contrabalançar o sedentarismo e assim evitar as doenças degenerativas associadas a ele. Em alguns casos são fornecidos exemplos de exercícios nos locais de trabalho, solicitando-se aos trabalhadores que desejarem, a realização dos exercícios-exemplo na situação de trabalho.

Addley, McQuillan \& Ruddley (2001) descrevem o programa de "Avaliação de Estilo de Vida e Prática de Atividades Físicas" promovido pelo governo irlandês no período de 1997 a 2000. Esse programa foi realizado junto aos funcionários públicos. As atividades se resumiam a uma avaliação dos participantes, realizada por enfermeiras fora dos locais de trabalho. Nessa avaliação, os funcionários que voluntariamente compareciam aos locais de avaliação respondiam a um questionário sobre seu nível de prática de exercícios físicos, seus hábitos alimentares e consumo de álcool e se eram fumantes ou não. A avaliação consistia em medidas fisiológicas da estatura, peso, índice de massa corporal, gordura, força, análises da urina, pressão sanguínea, colesterol e estamina, usando uma bicicleta ergométrica. Seis meses depois da avaliação, os pesquisadores solicitaram aos participantes que respondessem a um questionário, enviado pelo correio, sobre seu estilo de vida. O questionário mostrou uma série de mudanças no estilo de vida dos participantes no que se refere a hábitos alimentares e à prática de exercícios físicos. Os autores concluem que esse tipo de programa de incentivo a práticas saudáveis de alimentação e exercícios é uma estratégia importante para a melhoria das condições de saúde da população, salientando que o programa foi realizado a partir dos locais de trabalho.

No Brasil, são raros os programas de atividade física nas empresas no sentido de promover uma conscientização e um aumento da prática de exercícios, visando a melhoria da qualidade de vida dos trabalhadores e da população em geral. Dois desses programas são do conhecimento das autoras. Apesar das tentativas, bem intencionadas, de proporcionar maiores oportunidades de os trabalhadores se exercitarem fisicamente, oferecendo descontos em academias, ou até academias próximas dos locais de trabalho e outros tipos de incentivo, a participação não é muito constante. Shephard (1999), em sua revisão sobre o assunto, aponta a existência de evidências limitadas de mudanças nos hábitos de atividade física, melhoria da capacidade aeróbica e diminuição dos riscos de doenças coronárias relacionadas a esses programas nos outros países.

Note-se, no entanto, que esse tipo de programa nada tem a ver com a prática de ginástica laboral. Os programas de ginástica laboral, como veremos a seguir, pretendem contrabalançar a atividade muscular inerente ao próprio trabalho e assim, ser um fator de prevenção das doenças ocupacionais que atingem o sistema músculo-esquelético, isto é, LER/DORT. Esse tipo de programa é muito mais comum nas empresas brasileiras. É impressionante a quantidade de educadores físicos, fisioterapeutas e empresas especializadas em programas de ginástica laboral que aparecem em uma busca despretensiosa na Internet, ressalvando-se que isto acontece apenas nos "sites" brasileiros. 


\section{Histórico das Práticas de Atividade Física no Trabalho e Ginástica Laboral}

Os programas de atividade física no trabalho são relativamente antigos. Existem registros desse tipo de atividade em 1925 na Polônia, Bulgária, Alemanha Oriental, Holanda e Rússia, quando era chamada de ginástica de pausa (Cañete, 1996). Na mesma época, impulsionada pela cultura e tradição oriental, a prática de atividade física no trabalho foi implantada no Japão. Inicialmente era destinada a algumas atividades ocupacionais, mas após a Segunda Guerra Mundial foi difundida por todo o país. A grande propagação desses programas na cultura japonesa é atribuída à veiculação de um programa da rádio Taissô, que envolve uma tradicional ginástica rítmica, com exercícios específicos, acompanhados por música própria. Esse tipo de atividade acontece todas as manhãs, sendo transmitida pela rádio e praticada, não somente nas fábricas ou ambientes de trabalho no início do expediente, mas também nas ruas e residências. Em geral, os movimentos propostos são lentos e compassados e servem como uma preparação para as atividades diárias em casa ou no trabalho.

A federação de Rádio Taissô no Brasil coordena mais de 5000 praticantes ligados a 30 entidades em quatro estados: São Paulo, Rio de Janeiro, Paraná e Mato Grosso do sul. (Polito $\&$ Bergamaschi, 2002).

No Brasil, após algumas experiências isoladas durante os anos 70, houve um período em que essas práticas caíram no esquecimento. Polito e Bergamaschi (2002) atribuem essa queda à carência de resultados que servissem de base para a sua disseminação.

$\mathrm{Na}$ segunda metade da década de 80 , a prática de atividades físicas nos locais de trabalho, já na forma de ginástica laboral, se associa ao aparecimento das LER/DORT, pois foi nessa época, em 1987, que houve o reconhecimento oficial da então chamada doença dos digitadores. Nos anos 90, a GL teve sua grande explosão no Brasil, passando, inúmeras empresas, a introduzir a execução de exercícios em suas rotinas laborativas. Os propósitos são diversificados, mas, na maioria dos casos, o objetivo é a "prevenção" de LER/DORT.

\section{Tipos de Programas de Ginástica Laboral}

A GL é uma prática desenvolvida dentro da empresa que consiste em exercícios específicos realizados no próprio local de trabalho. Segundo Zilli (2002) a GL pode ser classificada de acordo com seu horário de aplicação:

Preparatória ou de Aquecimento: realizada no início da jornada de trabalho, ela ativa fisiologicamente o organismo, prepara para o trabalho físico e melhora o nível de concentração e disposição, elevando a temperatura do corpo, oxigenando os tecidos e aumentando a freqüência cardíaca. Tem a duração aproximada de 10 a 12 minutos. Inclui exercícios de coordenação, equilíbrio, concentração, flexibilidade e resistência muscular.

Compensatória: com duração de 5 a 10 minutos durante a jornada de trabalho, sua principal finalidade é compensar todo e qualquer tipo de tensão muscular adquirido pelo uso excessivo ou inadequado das estruturas musculoligamentares. Tem o objetivo de melhorar a circulação com a retirada de resíduos metabólicos, modificar a postura no trabalho, reabastecer os depósitos de glicogênio e prevenir a fadiga muscular. São sugeridos exercícios de alongamento e flexibilidade, respiratórios e posturais. 
Relaxamento: realizada no final da jornada de trabalho durante 10 ou 12 minutos, tem como objetivo a redução do estresse, alívio das tensões, redução dos índices de desavenças no trabalho e em casa, com conseqüente melhora da função social. São realizadas automassagens, exercícios respiratórios, exercícios de alongamento e flexibilidade e meditação.

A GL pode também ser classificada quanto ao seu objetivo:

Ginástica Corretiva ou Postural: relacionada com o equilíbrio dos músculos agonistas/antagonistas, isto é, alongamento dos músculos mais sobrecarregados e fortalecimento dos músculos em desuso ou em pouco uso. Sua execução pode durar entre 10 e 12 minutos, todos os dias ou três vezes por semana.

Ginástica de Compensação: tem o objetivo de evitar vícios posturais e o aparecimento da fadiga, principalmente por posturas extremas, estáticas ou unilaterais. Podem ser realizados movimentos simétricos de alongamento dentro do próprio setor ou ambiente de trabalho entre 5 a 10 minutos.

Existem ainda outras modalidades que se aproximam mais de um real programa de condicionamento físico, como por exemplo:

Ginástica Terapêutica: tem como objetivo o tratamento de distúrbios, patologias ou alterações posturais com grupos de funcionários avaliados previamente e separados por queixas. Tal modalidade é decorrente de um diagnóstico médico, em razão da objetividade do tratamento. É realizada em um local apropriado e não no local de trabalho. Sua duração pode chegar a 30 minutos.

Ginástica de Manutenção ou Conservação: é um programa de continuidade após obtenção do equilíbrio muscular alcançado pelas técnicas corretiva ou terapêutica citadas. Pode evoluir para um programa de condicionamento físico aeróbico associado a reforço muscular e alongamentos. Nesse caso é necessário que a empresa disponha de sala especial para o treinamento para que o funcionário possa utilizar seus horários de folga com duração de 45 a 90 minutos.

Os três primeiros tipos descritos, cuja combinação denominamos aqui de GL, são os mais utilizados nas empresas e mais propagandeados em panfletos e através da Internet. As propagandas prometem vários benefícios tanto para as empresas quanto para os funcionários. Os pontos ressaltados como benefícios são os mostrados no Quadro 1. A mesma lista com pequenas variações pode ser encontrada em vários "sites" de propaganda de ginástica laboral e em algumas publicações sobre a GL (Cenpre, 2004; Centro da Terra, 2004; Corpo e Saúde, 2004; Fundão, 2004; Ginástica.Net, 2004; Supernet, 2004; Centro de Cultura Oriente Ocidente, 2004; Pessoal.Onda, 2004; Viaseg, 2004; Razão do Todo, 2004; Centro Norte Clínica de Dor Crônica, Ortopedia e Reabilitação, 2004; Ergofisio, 2004; Insmed, 2004; Mesp, 2004; Porsports, 2004; Quavie, 2004; Reactive, 2004; Supporte, 2004; Planeta Terra, 2004). 
Quadro 1: Benefícios atribuídos à ginástica laboral nos "sites" da Internet (retirado de Saúde e Performance, 2004)

\begin{tabular}{|l|l|}
\hline Benefícios para as empresas: & Benefícios para o trabalhador: \\
\hline Redução do índice de absenteísmo & Fisiológicos \\
Maior proteção legal & Prevenir a DORT/LER \\
Aumento dos lucros & Prevenir lesões \\
$\begin{array}{l}\text { Diminuição dos acidentes de trabalho, com prevenção } \\
\text { de doenças profissionais }\end{array}$ & $\begin{array}{l}\text { Diminuir tensões generalizadas e } \\
\text { relaxar }\end{array}$ \\
Reflexão na capacidade de produção / produtividade & Amenizar fadiga muscular e emocional \\
Integração dos trabalhadores & Prevenir o estresse \\
Baixo custo de implantação do programa & Melhorar a postura \\
Funcionamento da ação como política de RH & Melhorar a condição do estado de saúde geral \\
Acompanhamento do médico da empresa ou RH & Psicológicos \\
Reduzir acidentes de trabalho e/ou afastamento do & Reforçar a auto-estima \\
trabalhador da empresa & $\begin{array}{l}\text { Aumento da capacidade de concentração } \\
\text { no ambiente de trabalho }\end{array}$ \\
& Conquista do momento destinado a ele \\
& Valorização do funcionário (homem / profissional) \\
& Sociais \\
& $\begin{array}{l}\text { Melhorar o relacionamento interpessoal } \\
\text { Melhorar a comunicação interna }\end{array}$ \\
& Participação ativa nas palestras debates e dinâmica \\
& \\
& \\
&
\end{tabular}

\section{Crescimento dos Programas de Ginástica Laboral no Ceará}

Para verificar a incidência dos programas de GL no estado do Ceará, foi realizado um levantamento junto a duas instituições que normalmente implantam esses programas em fábricas ou empresas de serviço. Procuramos verificar o ano da implantação, bem como o tipo de profissional responsável pelo desenvolvimento e manutenção do programa.

A Tabela 1 mostra o número de empresas que iniciaram programas de GL durante o período do levantamento. Nota-se na tabela o aumento do número de empresas que têm adotado esses programas.

A Tabela 2 mostra o tipo de profissional responsável pela implantação e manutenção dos diversos programas. Nota-se pela tabela que há a uma certa variedade no tipo de profissional que realiza os programas e que, em alguns casos, a manutenção do programa é deixada a cargo de multiplicadores, isto é, empregados da empresa treinados e instados a dirigir os exercícios nas pausas programadas. 
Tabela 1: Número de empresas que implantaram programas de GL no Ceará

\begin{tabular}{|c|c|}
\hline Ano da Implantação & Número de Empresas \\
\hline 1998 & 1 \\
\hline 1999 & 5 \\
\hline 2000 & 3 \\
\hline 2001 & 4 \\
\hline 2002 & 7 \\
\hline 2003 & 8 \\
\hline 2004 (até abril) & 6 \\
\hline Total & 34 \\
\hline
\end{tabular}

Tabela 2: Tipo de profissional responsável pela implantação e manutenção dos programas de GL

\begin{tabular}{|c|c|}
\hline Tipo de Profissional & Freqüência \\
\hline Educador Físico & 16 \\
\hline Fisioterapeuta & 2 \\
\hline Estagiários Educação Física & 12 \\
\hline Multiplicadores & 7 \\
\hline Total & 37 \\
\hline
\end{tabular}

Em nenhum dos programas pesquisados há um acompanhamento individual ou acompanhamento médico dos empregados no que se refere à sua real capacidade de realizar a atividade proposta e, em nenhum dos casos encontrou-se o tipo de ginástica classificada como Terapêutica ou de Manutenção.

\section{Eficácia dos Programas de GL}

Ao discutir o problema da eficácia dos programas de GL é importante separar seus vários objetivos e metas, bem como a forma com que o programa é aplicado.

\subsection{A não-comprovação da prevenção de LER/DORT pela GL}

Encabeçando a lista dos efeitos benéficos temos a diminuição das LER/DORT (Quadro 1). No entanto, os estudos que comprovam algum tipo de eficácia dos programas de GL, contêm os três primeiros tipos de GL descritos acima: ginástica preparatória (10 a 12 minutos), ginástica compensatória (5 a 10 minutos) e ginástica de relaxamento (10 a 12 minutos), que se constitui no programa de GL mais comum em voga nas empresas. Entretanto, são raros.

Dishman, Oldenburg, O'Neal e Shephard (1998) realizaram uma meta-análise das pesquisas que investigaram a eficiência de programas de práticas físicas, sejam programas de incentivo a atividades físicas, sejam programas de GL. O estudo investigou 73 pesquisas e verificou que, no caso de intervenções tipo GL, há alguns indícios de melhoria da condição física dos participantes, mas os benefícios são pequenos. Além disso, os autores afirmam que há poucas evidências mostrando que uma intervenção tipo GL produz um aumento na prática de atividades físicas de seus participantes. Por outro lado, há evidências de que os empregados preferem não realizar exercícios físicos no trabalho. De uma maneira geral, os autores concluem que os programas de intervenção nos locais de trabalho ainda não 
demonstraram um efeito positivo sobre o incentivo de práticas de atividades físicas e sobre as condições físicas dos empregados.

Sommerich e Joines (1999) concluem, em seu artigo sobre programas de prevenção de LER/DORT, que embora uma boa forma física e a prática de atividades físicas sejam geralmente aceitas como um meio de reduzir as LER/DORT, a literatura não fornece evidências claras para essa suposição. A literatura sobre a medicina do esporte, no entanto, indica que esportes que envolvem atividades de natureza repetitiva ou que envolvem esforço (tais como o tênis e o baseball) estão relacionados ao aparecimento de afecções no sistema músculo-esquelético, ao invés de sua prevenção. Complementam sua afirmação dizendo que é interessante notar que nas atividades esportivas profissionais os jogadores fazem um número maior de pausas para recuperação e a duração das tarefas intensas é bem menor do que ocorre nos locais de trabalho tradicionais, onde os trabalhadores devem realizar trabalhos repetitivos e cansativos por períodos de 8 horas durante 5 ou 6 dias na semana.

Na mesma linha, o trabalho do Painel sobre Desordens Musculoesqueléticas do Governo dos Estados Unidos (National Research Council and Institute of Medicine, 2001) afirma que são poucos os estudos que relacionam programas de ginástica laboral e a prevenção da LER/DORT. Os que existem possuem um rigor científico limitado e não são generalizáveis. A conclusão é que os resultados são controversos, com alguns estudos mostrando uma correlação positiva entre ausência de sintomas e a prática da GL e outros mostrando o contrário.

Em uma revisão mais recente sobre a eficácia da ginástica laboral na prevenção de LER/DORT. Embora sugiram que os exercícios melhorem a saúde e diminuam a severidade e o custo do tratamento das lesões, Hess e Hecker (2003) concluem que os poucos estudos específicos sobre os programas de ginástica laboral não fornecem evidências conclusivas nem a favor nem contra a ginástica laboral, confirmando revisões anteriores sobre o mesmo assunto.

No Brasil existem alguns documentos e textos que alegam trazer indicações seguras de que a GL aumenta a prática de atividades físicas dos funcionários e diminui a incidência de dores e demais sintomas relacionados a LER/DORT. Um deles afirma que:

A ginástica laboral adaptada para as necessidades impostas pelo tipo de trabalho, realizada sem sair do posto, em breves períodos de tempo, ao longo de todo o dia de trabalho, pode produzir resultados positivos para os funcionários e para a empresa (Pinto $\&$ Souza, 2004).

A referência fornecida para corroborar essa afirmação nos leva ao "site" do SESC na Internet, um das instituições que mais tem oferecido programas desse tipo, ao lado do SESI e do SENAI. No entanto, não há de fato um estudo científico sobre o assunto realizado por essas instituições que comprove a afirmação de que a GL diminui as afecções relacionadas a LER/DORT. Apesar disso, essas instituições continuam propondo e implantando programas de ginástica laboral nos locais de trabalho, aparentemente com grande sucesso.

Martins e Martins (2000) realizaram um estudo junto a 90 funcionários da Justiça Federal de Santa Catarina. Após 27 sessões de GL, os participantes responderam a um questionário auto-administrado. A conclusão foi a de que os dados sugerem uma boa aceitação do programa pelos funcionários e que a GL parece ter sido eficaz na prevenção de LER/DORT. No entanto, a pesquisa não apresenta nenhum dos controles habituais para esse tipo de pesquisa: não há um grupo controle e nem mesmo o controle do próprio sujeito (aplicação do mesmo questionário antes-depois). Não foram realizadas medidas ou exames clínicos que possam comprovar as sugestões das autoras. Resta o fato da boa aceitação por parte dos participantes e o auto-relato da diminuição dos sintomas relacionados a dores no corpo. 
Uma das autoras, Martins (2000), realizou um estudo com 26 trabalhadores da reitoria da Universidade Federal de Santa Catarina onde foram medidas a flexibilidade, a força, o percentual de gordura e a pressão arterial dos participantes. Após 54 sessões de ginástica laboral, realizadas três vezes por semana durante 15 minutos, encontrou-se uma melhoria significante do percentual de gordura, da pressão arterial, da flexibilidade medida pelo teste de "Sentar e Alcançar". Obteve-se também um aumento de amplitude da abdução do ombro, da hiperextensão do cotovelo, da flexão do punho, da extensão do quadril, da flexão e da hiperextensão do joelho. No entanto, os resultados não mostraram uma correlação positiva entre a freqüência às sessões de exercícios nos locais de trabalho e os benefícios apontados nas mensurações realizadas. Além disso, a pesquisa não controlou outros fatores como a prática de exercícios, esportes e atividades físicas fora do trabalho. A autora relata que 38\% da amostra em questão realizava esse tipo de atividade antes e durante a implantação do programa. Na amostra estudada havia apenas um caso de LER/DORT. Por outro lado, parece ter havido uma intensificação da prática de exercícios fora do trabalho devido ao programa, o que explicaria as melhorias encontradas.

Em uma outra investigação sobre o assunto, Longen (2003) analisou a implantação e continuidade de um programa de GL em uma única empresa ao longo do tempo e concluiu que houve uma grande redução nos casos de LER/DORT nos dois anos iniciais do programa, mas notou que os casos voltaram a crescer depois desse período. O fenômeno é explicado em termos do significado do programa para a empresa e para os funcionários, uma espécie de "efeito Hawthorne" como será explicado a seguir.

Assim, até o momento não existem estudos conclusivos mostrando uma relação positiva entre a prática da GL e o não aparecimento ou melhoria da LER/DORT.

\subsection{Dúvidas sobre a eficácia de exercícios de alongamento e aquecimento}

Alguns estudos na literatura apontam a não existência de evidências científicas sobre os efeitos positivos do alongamento na fase de aquecimento para algumas atividades esportivas. Knudson (1999), revendo o assunto, afirma que os exercícios de alongamento aumentam a tolerância em relação aos movimentos amplos, temporariamente, mas não influenciam a musculatura, que seria o efeito esperado para a prevenção de lesões nos músculos.

O British Medical Journal em seu editorial de agosto de 2002 refere-se à mesma questão, apontando a falta de evidências científicas sobre o benefício dos exercícios de alongamento para a musculatura e para a prevenção de lesões. Herbert e Gabriel (2002) realizaram o estudo que serve de embasamento para o editorial e a conclusão dos autores é que o alongamento antes ou depois dos exercícios físicos não promove a prevenção de distenções musculares nem previne lesões musculares de atletas. trabalho?

O que não dizer de exercícios de alongamento e aquecimento para as atividades de

\section{3. "Efeito Hawthorne"}

Elton Mayo e colegas (Mayo, 1946), um pouco antes da Segunda Guerra Mundial, realizaram um experimento em uma fábrica nos Estados Unidos e descobriram que os trabalhadores mudavam seu desempenho e sua morale pelo simples motivo de perceberem uma atenção maior por parte da gerência em relação à sua vivência no trabalho. Esse efeito é conhecido até hoje como efeito Hawthorne. A ginástica laboral parece repetir o efeito 
Hawthorne, de tal maneira que os trabalhadores se sentem melhores ao participarem voluntariamente dos programas de GL.

Em um contexto ligeiramente diferente, mas relacionado, existem evidências que comprovam uma melhora na saúde mental daqueles indivíduos que se engajam em atividades físicas (Martin \& Wade, 2000). Atividades físicas têm sido utilizadas até mesmo em contextos terapêuticos, como uma ajuda nas terapias psicológicas tradicionais, mostrando efeitos positivos no aumento da auto-estima, humor, estabilidade e histamina, na redução da ansiedade, neuroticismo e estresse, bem como melhoria de quadros depressivos leves ou moderados (Chung \& Baird, 1999). É claro que, nesses casos, o tipo de programa de ginástica ou atividade física proposto é bem mais duradouro e contínuo do que os propostos nos programas de GL.

\subsection{Pausas}

Em um estudo sobre a prevenção de LER/DORT, Galinsky, Swanson, Sauter, Hurell \& Schleifer (2000) mostraram a importância das pausas para a prevenção das afecções. Os autores compararam dois esquemas de pausas, sendo que no segundo esquema havia o dobro de pausas do que no primeiro. Eles concluíram que quando há um número maior de pausas curtas durante a jornada de trabalho, os trabalhadores sentem menos dores, além de se sentirem mais dispostos.

Transpondo-se os resultados desse estudo para as situações de trabalho onde há programas de GL, estes programas, sem dúvida, permitem que os trabalhadores façam um número maior de pausas, onde antes não havia nenhuma. Este fato pode ser uma explicação para os resultados positivos obtidos por Martins e Martins (2000) e Martins (2000).

\section{Efeitos benéficos para as empresas}

O que chama a atenção na lista dos efeitos benéficos para as empresas, anteriormente apresentada, é a frase "maior proteção legal" e o conceito de "prevenção" proposto.

As instituições e profissionais que implantam os programas de GL, às vezes denominam esses programas de ergonomia ou prevenção ergonômica das LER/DORT. Pelo que se descreveu acima, fica evidente que os programas de GL não visam uma melhoria das condições, do ambiente ou da organização do trabalho, objetivo primeiro das intervenções ergonômicas. Pelo contrário, os programas de GL pretendem intervir diretamente no sistema músculo-esquelético dos trabalhadores, fortalecendo-o ou compensando pelos movimentos que ocorrem durante o trabalho. Nesse sentido, os objetivos da ergonomia e da GL são distintos e os meios utilizados completamente diferentes. Assim, fica claro que os programas de ginástica laboral não podem e não devem ser confundidos com intervenções ergonômicas, pois há uma diferença fundamental de objetivos e meios aplicados nas suas respectivas intervenções. Deste modo, as instituições que se referem aos seus serviços de ginástica laboral como sendo uma intervenção ergonômica estão, de certa forma, faltando com a verdade. A definição de ergonomia proposta pela "International Ergonomics Association" (IEA) inclui o seguinte trecho:

Os praticantes da Ergonomia, Ergonomistas, contribuem para o planejamento, projeto e a avaliação de tarefas, postos de trabalho, produtos, ambientes e sistemas para torná-los compatíveis com as necessidades, habilidades e limitações das pessoas (Associação Brasileira de Ergonomia, 2004). 
De acordo com Mike Wynn (2004) ${ }^{3}$ que participa da lista internacional de discussão de Ergonomia (ERGOWEB-LIST):

...exercícios não se adequam à definição de controles técnicos (modificações no trabalho para redução de riscos) e tampouco nos controles administrativos (modificações no gerenciamento e políticas para redução à exposição aos riscos), os métodos aceitos para o controle das LER/DORT.

Ao contrário dos programas de GL, as intervenções ergonômicas têm se mostrado efetivas na redução e prevenção da incidência das LER/DORT. Talvez esse seja um dos motivos de se tentar manter a confusão entre programas de GL e programas de ergonomia.

O outro motivo pode estar relacionado ao fato de os empregadores estarem obrigados a seguir as Normas Regulamentadoras do Trabalho (NRs), entre elas a NR17: Ergonomia. A idéia da "proteção legal" é a de que os programas de GL poderiam servir como substitutos da Análise Ergonômica do Trabalho e das intervenções daí decorrentes, como preconizado pela NR17. No entanto, em nenhum momento, em seus artigos, a NR17 se refere a programas de ginástica laboral como uma exigência legal na prevenção de LER/DORT. Aliás, a NR17 não se refere de modo algum a programas de ginástica laboral.

A promessa de "maior proteção legal" tem levado algumas empresas a tornar a participação nos programas de GL obrigatória e, em algumas delas, o funcionário assina um documento onde atesta estar ciente da necessidade de realizar a ginástica laboral. Neste caso, a empresa está utilizando a participação nos programas de GL como uma proteção contra futuros processos trabalhistas relacionados a LER/DORT. A idéia é a de que, se o funcionário não participar do programa, ele está, conscientemente, colocando sua saúde em risco. Ao invés de promover mudanças nas condições de trabalho que se constituem em agravos à saúde dos trabalhadores, parece mais fácil e mais "econômico" (como propõe a lista de benefícios para as empresas), implantar paliativos. O caso da GL é ainda mais grave, porque a GL, até o momento, não comprovou sua eficácia na prevenção da LER/DORT.

Por outro lado, não há estudos que comprovem a diminuição do absenteísmo e da maior disponibilidade para o trabalho. No entanto, alguns dos estudos analisados acima apontam um melhor clima organizacional e satisfação com as condições de trabalho, bem no estilo do efeito Hawthorne.

\section{Desvantagens dos Programas de GL para os trabalhadores}

As desvantagens dos programas de GL estão relacionadas principalmente com as implicações de sua aplicação. Uma delas é a inversão na relação de causa e efeito das doenças ocupacionais: não são as condições de trabalho que estão inadequadas, mas sim o trabalhador, que não possui um sistema músculo-esquelético forte o suficiente para dar conta das condições de trabalho. Essa idéia é a que está por trás daquelas situações onde a ginástica é obrigatória e o trabalhador é obrigado a assinar um "termo de compromisso". Esse termo de compromisso tem sido utilizado, na prática, como um documento que isenta a empresa da responsabilidade pela ocorrência da doença ocupacional, caso ela venha a ocorrer.

A inversão da relação de causa e efeito é grave, pois pode levar a um sentimento de inadequação do trabalhador. Em outros setores da gestão atual das empresas, a tentativa é fazer com que o trabalhador assuma todas as responsabilidades pelo desempenho, pela qualidade e, no caso da GL, pelo seu condicionamento físico. O seu estado físico deve ser de

3 ERGOWEB-LIST, 5 de Abril de 2004. Disponível em www.ergoweb.com. 
tal ordem a permitir que ele trabalhe sob condições estressantes, com ritmos acelerados e poucos momentos para relaxamento, sem se cansar e sem adoecer.

\subsection{Inadequação dos programas oferecidos}

Além disso, alguns desconfortos estão associados à realização da GL no local de trabalho. Em primeiro lugar, é muito desconfortável realizar exercícios físicos com as roupas de trabalho, principalmente em um país tropical e para aqueles trabalhadores que não utilizam uniformes de trabalho ou não efetuam troca de roupas para trabalhar.

Um segundo ponto relaciona-se com o local onde a GL é realizada. Sem dúvida, o local de trabalho não é apropriado para a prática de atividades físicas, pois são locais de trabalho e não de recreação ou lazer. Em geral, são locais que não possuem espaço suficiente para a realização dos exercícios: ambientes fechados e, às vezes, sem ventilação.

Um terceiro ponto se refere ao constrangimento imposto ao funcionário que deve realizar os exercícios na frente dos colegas e, às vezes, de seus chefes imediatos.

Em alguns casos, os programas de GL são gerenciados por multiplicadores como mostra a Tabela 2. Nesses casos, não há um acompanhamento eficaz na diferenciação dos exercícios propostos para se adequarem aos diferentes biótipos, ritmos e características dos empregados. Dependendo do tipo de treinamento oferecido aos multiplicadores podem ocorrer falhas no acompanhamento da realização dos exercícios.

\section{Conclusões}

Os vários estudos revisados apontam os aspectos positivos da prática de exercícios físicos na prevenção de doenças coronárias e outras doenças degenerativas, bem como influências positivas sobre o bem estar psicológico e social dos indivíduos que praticam algum tipo de esporte ou atividade física. A vida moderna tem levado a maioria das pessoas a uma vida sedentária com poucas atividades físicas, o que torna a prática de exercícios necessária para a promoção da saúde. O combate ao sedentarismo é, portanto, um problema de saúde pública e deve ser abordado pelas instituições governamentais de saúde. Por outro lado, a prática de atividades físicas pode e deve ser incentivada a partir do trabalho, uma vez que esse canal atinge um grande número de indivíduos. Apesar da pouca efetividade desses programas, segundo os estudos revisados, eles continuam sendo uma proposta interessante e podem produzir efeitos positivos no combate ao sedentarismo e suas conseqüências.

Deve-se entender a prática de exercícios físicos como benéfica quando realizada pela livre vontade dos indivíduos, em situações de sua escolha, sem constrangimentos, por períodos de pelo menos três vezes por semana com duração de aproximadamente uma hora cada sessão.

Bastante diversa é a maneira como comumente se desenrolam os programas de ginástica laboral. Os programas propõem atividades obrigatórias, em situações inadequadas e por períodos de curta duração (10 a 12 minutos cada sessão). Além disso, não existem evidências conclusivas sobre a efetividade dos programas de GL, nem como incentivo a prática de esportes, nem como um método de promoção da saúde nos locais de trabalho. Algumas evidências positivas existem, mas são raras e pouco generalizáveis, o que não justifica o grande número de empresas que vem empregando esse método na prevenção de LER/DORT. 
Em contraposição a esse pequeno efeito existem os benefícios que a empresa pode obter ao inverter a relação de causa e efeito das condições de trabalho sobre a saúde dos trabalhadores. Para as empresas é vantajosa a implantação de programas de GL na medida em que essa ação pode ser convertida em prova nos processos trabalhistas. Por outro lado, os programas de GL, embora em alguns casos se afirme que devam vir acompanhados de análises ergonômicas das atividades e mudanças nas condições de trabalho, parecem ser uma maneira mais econômica e mais fácil, no pensamento de alguns empresários, de lidar com o problema das LER/DORT, apesar de pouco efetivos.

O artigo de Belfort (2003) publicado no Jornal do Commércio de Pernambuco ostenta o sugestivo título "Ginástica laboral traz benefícios à produção". No artigo, um representante do SESI afirma que a procura por programas de ginástica laboral está aumentando: "esse aumento ocorre porque as companhias perceberam que o funcionário feliz passa a trabalhar com mais satisfação e isso também traz resultados positivos para o processo produtivo". No mesmo artigo há depoimentos de gerentes de várias empresas atestando a boa rentabilidade econômica e produtiva dos programas de ginástica laboral. Por outro lado, são poucas as referências sobre a melhoria da saúde dos funcionários e não há qualquer alusão à melhoria das condições de trabalho.

Diante das evidências encontradas na literatura e da constatação do crescimento da implantação de programas de ginástica laboral no Brasil, acreditamos que é necessário repensar quem está realmente se beneficiando dos programas de ginástica laboral e para que eles realmente estão servindo.

\section{Referências}

Associação Brasileira de Ergonomia (2004). Definição internacional de ergonomia. Internet: www.abergo.org.br. Acesso em 02/2004.

Addley, K., McQuillan, P. \& Ruddley, M. (2001). Creating healthy workplaces in Northern Ireland: evaluation of a life style and physical activity assessment programme. Occupational Medicine, 51 (7), 439-449.

Belfort, A. (2003, 20 de julho). Ginástica laboral traz benefícios à produção. Jornal do Commércio de Pernambuco. Internet: www.jc.uol.com.br. Acesso em 02/2004.

Cañete, I. (1996). Humanização: desafio da empresa moderna - a ginástica laboral como um novo caminho. Porto Alegre: Foco.

Cenpre (2004). Ginástica laboral. Internet: www.cenpre.com.br/cont_ginastica.html. Acesso em $10 / 2004$.

Center for Disease Control and Prevention (2004). Healthy People 2010. Internet: www.healthypeople.gov. Acesso em 04/2004.

Centro da Terra (2004). Ginástica laboral. Internet: www.cdaterra.com.br/glab.htm. Acesso em $10 / 2004$.

Centro de Cultura Oriente Ocidente (2004). Ginástica laboral. Internet: www.falconi.com.br/ginastica_laboral.htm. Acesso em 10/2004.

Centro Norte Clínica de Dor Crônica, Ortopedia e Reabilitação (2004). Ginástica laboral. Internet: www.clinicacentronorte.com.br/ginastica\%20e\%20quick.htm. Acesso em 10/2004.

Chung, Y. B. \& Baird, M. K. (1999). Physical exercises as a counseling intervention. Journal of Mental Health Counseling, 21 (2), 124-135. 
Codo, W. (1998). Providências na organização do trabalho para a prevenção da LER. In W. Codo \& M. C. C. G. Almeida (Org.). Lesões por Esforços Repetitivos: Diagnóstico, Tratamento e Prevenção: uma abordagem multidisciplinar. Petrópolis: Vozes.

Corpo e Saúde (2004). Ginástica laboral. Internet: www.corposaude.com.br/laboral.htm. Acesso em $10 / 2004$.

Craig, C. L., Brownson, R. C., Cragg, S. E. \& Dunn, A. L. (2002). Exploring the effect of the environment on physical activity: a study examining walking to work. American Journal of Preventive Medicine, 23 (2S), 36-43.

Dishman, R. K., Oldenburg, B., O’Neal, H. \& Shephard, R. (1998). Worksite Physical Activity Interventions. American Journal of Preventive Medicine, 15 (4), 344-361.

Ergofisio (2004). Ginástica laboral. Internet: www.ergofisio.com.br/ginasticalaboral.htm. Acesso em $10 / 2004$.

Fundão (2004). Ginástica laboral. Internet: www2.fundao.pro.br/articles.asp?cod=57. Acesso em $10 / 2004$.

Galinsky, T. L., Swanson, N. G., Sauter, S. L., Hurell, J. J. \& Schleifer, L. M. (2000). A field study of supplementary rest breads for data-entry operators. Ergonomics, 43 (5), 622-638.

Ginástica.net (2004). Ginástica laboral. Internet: www.ginastica.net/laboral.html. Acesso em $10 / 2004$.

Herbert, R. D. \& Gabriel, M. (2002). Effects of stretching before and after exercising on muscle soreness and risk of injury: systematic review. British Journal of Medicine, 325, 468-470.

Hess, J. \& Hecker, S. (2003). Stretching at work for injury prevention: issues, evidence, and recommendations. Applied Occupational and Environmental Hygiene, 18 (5), 331-338.

Insmed (2004). Ginástica laboral. Internet: www.insmed.com.br/programacao. Acesso em 10/2004.

Knudson, D. (1999). Stretching during warm-up: do we have enough evidence? Journal of Physical Education Recreation and Dance, 70 (7), 24-27.

Lampl, M., Wooley, S., Conine, L., Hoehne, L. \& Moorman, L. (2000). The effectiveness of an ergonomic injury management (EIM) process. Proceedings of the IEA 2000 Congress, San Diego, California, 5, 382.

Longen, W. C. (2003). Ginástica Laboral na Prevenção de LER/DORT? Um Estudo Reflexivo em uma Linha de Produção. Dissertação de Mestrado, Engenharia de Produção, Universidade Federal de Santa Catarina. Santa Catarina. Internet: teses.eps.ufsc.br/defesa/pdf/8426.pdf. Acesso em 10/2004.

Maciel, R. H. (1998). Ergonomia e lesões por esforços repetitivos. In W. Codo \& M. C. C. G. Almeida (Org.). Lesões por Esforços Repetitivos: Diagnóstico, Tratamento e Prevenção: uma abordagem multidisciplinar. Petrópolis: Vozes.

Martin, J. C. \& Wade, T. J. (2000). The relationship between physical exercise and distress in a national sample of Canadians. Canadian Journal of Public Health, 91 (4), 302-306.

Martins, C. O. e Martins, M. O. (2000). Eficácia da ginástica laboral na prevenção aos DORT e sua aceitação por funcionários públicos de Florianópolis-SC. Anais do XXIII Simpósio Internacional de Ciências do Esporte - Atividade Física, Fitness e Esporte (p. 173), São Paulo.

Martins, C. O. (2000). Efeitos da Ginástica Laboral em Servidores da Reitoria da UFSC. Dissertação de Mestrado, Engenharia de Produção, Universidade Federal de Santa Catarina, Santa Catarina. Internet: www.eps.ufsc.br. Acesso em 10/2004.

Mayo, E. (1946). Problemas Humanos de uma Civilización Industrial. Buenos Aires: Ed. Galatea-Nueva Visión.

Mesp (2004). Ginástica laboral. Internet: www.mesp.com.br/informesp/dicas\%2003.htm. Acesso em $10 / 2004$. 
Ministério da Saúde, Brasil (2000). Protocolo de investigação, diagnóstico, tratamento e prevenção de LER/DORT. Brasília: Ministério da Saúde.

National Research Council and the Institute of Medicine (2001). Musculoskeletal disorders and the workplace: low back and upper Extremities. Panel on Musculoskeletal Disorders and the Workplace. Commission on Behavioral and Social Sciences and Education. Washington, DC: National Academy Press.

Pessoal.Onda (2004). Ginástica laboral. Internet: www.pessoal.onda.com.br/kikopers/ ginastica_laboral.htm. Acesso em 10/2004.

Pinto, A. C. C. S. \& Souza, R. C. P. (2004). A Ginástica laboral como ferramenta para a melhoria da qualidade de vida no setor de cozinha em restaurantes. Internet: www.eps.ufsc.br. Acesso em 10/2004.

Planeta Terra (2004). Ginástica laboral. Internet: www.planeta.terra.com.br/saude/ginlabor/ capa.html. Acesso em 10/2004.

Polito, E. \& Bergamaschi, E. C. (2002). Ginástica Laboral: teoria e prática. Rio de Janeiro: Sprint.

Prosports (2004). Ginástica laboral. Internet: www.prosports.esp.br/links/gl.php. Acesso em 10/2004.

Puska, P., Helasoja, V., Prätälä, R., Kasmel, A. \& Klumbiene, J. (2003). Health behavior in Estonia, Finland and Lithuania: 1994-1998. European Journal of Public Health, 13, 11-17.

Quavie (2004). Ginástica laboral. Internet: www.quavie.com.br/produtos/GinasticaLaboral.htm. Acesso em 10/2004.

Ranney, D. (2000). Distúrbios Osteomusculares Crônicos Relacionados ao Trabalho. São Paulo: Roca.

Razão do todo (2004). Ginástica laboral. Internet: www.razaodotodo.com.br. Acesso em 10/2004.

Reactive (2004). Ginástica laboral. Internet: www.reactive.com.br/ginastica.htm. Acesso em 10/2004.

Rios, R. P. (1998). LER (Lesões por Esforços Repetitivos) Ciência e Lei - Novos Horizontes da Saúde e do Trabalho. Belo Horizonte: Health, 1998.

Saúde e Performance (2004). Ginástica laboral. Internet: www.saudeperformance.com.br/ ginastica_laboral.php. Acesso em 10/2004.

Shephard, R. J. (1999). Do Work-Site Exercise and Health Programs Work? The Physician and Sports Medicine Online. Internet: www.physsportsmed.com/issues/1999/02_99/shephard.htm. Acesso em $10 / 2004$

Sommerich, C. M. \& Joines, S. M. B. (1999). Review of the literature with respect to the work relatedness of musculoskeletal disorders of the neck and upper back. Request by the U.S. Department of Labor's Occupational Safety and Health Administration in support of the development of the ergonomics work standard. Internet: www.osha-slc.gov/ergonomics-standard/PROPOSED/heapp1.html, at section B, titled Epidemiology of Neck and Upper-Back Musculoskeletal Disorders. Acesso em 03/2000.

Souza, M. F. M. (1999). Um estudo de prevalência e risco para as lesões por esforços repetitivos (LER/DORT) entre trabalhadoras e trabalhadores em processamento de dados bancários. Tese de Doutorado, Faculdade de Medicina, Universidade de São Paulo, São Paulo.

Supernet. (2004). Fisiologia do trabalho: ginástica laboral. Internet: www.supernet.com.br/ med/ergo_ler/fisiot_trabalho.html. Acesso em 10/2004.

Supporte (2004). Ginástica laboral. Internet: www.supporte.ind.br/laboral.htm. Acesso em 10/2004.

Titze, S., Martin, B. W., Seiler, R., Stronegger, W. \& Marti, B. (2001). Effects of a lifestyle physical activity intervention on stages of change and energy expenditure in sedentary employees. Psychology of Sport and Exercise, 2, 103-116.

Verthein, M. A. R. \& Gomez, C. M. (2001). As armadilhas: bases discursivas da neuropsiquiatrização das LER. Ciência e Saúde Coletiva, 6 (2), 457-470.

Viaseg (2004). Ginástica laboral. Internet: www.viaseg.com.br/glaboral.htm. Acesso em 10/2004. 
Yancey, A. K., McCarthy, W. J., Taylor, W. C., Merlo, A., Gewa, C., Weber, M. D. \& Fielding, J. E. (2004). The Los Angeles Lift Off: a sociocultural environmental change intervention to integrate physical activity into the workplace. Preventive Medicine, 38 (6), 848-856. Internet: www.elsevier.com/locate/ypmed. Acesso em 10/2004.

Zilli, C. M. (2002). Manual de cinesioterapia/Ginástica Laboral, Uma Tarefa Interdisciplinar com Ação Multiprofissional. Curitiba: Lovise.

Endereço para correspondência: rhmaciel@rapix.com.br 